\title{
LONG-TERM VARIATION IN THE SUN'S ACTIVITY CAUSED BY MAGNETIC ROSSBY WAVES IN THE TACHOCLINE
}

\author{
Teimuraz V. Zaqarashvili ${ }^{1,5}$, Ramon Oliver $^{2}$, Arnold Hanslmeier ${ }^{3}$, Marc Carbonell $^{4}$, \\ Jose Luis Ballester ${ }^{2}$, Tamar Gachechiladze ${ }^{5}$, and Ilya G. Usoskin ${ }^{6}$ \\ ${ }^{1}$ Space Research Institute, Austrian Academy of Sciences, Schmiedlstrasse 6, 8042 Graz, Austria; teimuraz.zaqarashvili@oeaw.ac.at \\ ${ }^{2}$ Departament de Física, Universitat de les Illes Balears, E-07122 Palma de Mallorca, Spain \\ ${ }^{3}$ Institute für Physik, Geophysik Astrophysik und Meteorologie, University of Graz, Univ.-Platz 5, 8010 Graz, Austria \\ ${ }^{4}$ Departament de Matemàtiques i Informàtica, Universitat de les Illes Balears, E-07122 Palma de Mallorca, Spain \\ ${ }^{5}$ Abastumani Astrophysical Observatory at Ilia State University, Tbilisi, Georgia \\ ${ }^{6}$ Sodankylä Geophysical Observatory and ReSoLVE Centre of Excellence, University of Oulu, FI-90014 Oulo, Finland \\ Received 2015 March 17; accepted 2015 May 6; published 2015 May 27
}

\begin{abstract}
Long-term records of sunspot number and concentrations of cosmogenic radionuclides (10Be and 14C) on the Earth reveal the variation of the Sun's magnetic activity over hundreds and thousands of years. We identify several clear periods in sunspot, $10 \mathrm{Be}$, and $14 \mathrm{C}$ data as $1000,500,350,200$, and 100 years. We found that the periods of the first five spherical harmonics of the slow magnetic Rossby mode in the presence of a steady toroidal magnetic field of 1200-1300 G in the lower tachocline are in perfect agreement with the timescales of observed variations. The steady toroidal magnetic field can be generated in the lower tachocline either due to the steady dynamo magnetic field for low magnetic diffusivity or due to the action of the latitudinal differential rotation on the weak poloidal primordial magnetic field, which penetrates from the radiative interior. The slow magnetic Rossby waves lead to variations of the steady toroidal magnetic field in the lower tachocline, which modulate the dynamo magnetic field and consequently the solar cycle strength. This result constitutes a key point for long-term prediction of the cycle strength. According to our model, the next deep minimum in solar activity is expected during the first half of this century.
\end{abstract}

Key words: Sun: activity - Sun: interior - Sun: oscillations

\section{INTRODUCTION}

Solar activity, which is manifested through huge energy releases in terms of coronal mass ejections and solar flares, basically determines the plasma conditions of near-Earth and interplanetary space, and possibly influences Earth's climate (Haigh 2007). Therefore, the prediction of solar activity is of paramount importance for our future scientific-technical strategy. The activity undergoes 11 years oscillations known as Schwabe cycles (Schwabe 1844) generally interpreted in terms of dynamo models (Charbonneau 2005). However, longterm records of sunspot number (Gleissberg 1939; Hill 1977) and concentrations of cosmogenic radionuclides on the Earth (Suess 1980; Stuiver \& Braziunas 1989; Solanki et al. 2004; Usoskin et al. 2004; Steinhilber et al. 2012) reveal the variation of the Sun's magnetic activity over hundreds and thousands of years.

The observed long-term variation in solar activity is still a mystery (Hathaway 2010). Three different explanations for the variation have been proposed. The first one suggests that the nonlinear solar dynamo permits a transition from periodic oscillations to chaotic behavior with a long period modulation of magnetic field strength (Weiss et al. 1984). The second mechanism considers random fluctuations in the dynamo parameters, which may mimic the main features of the longterm variations in the activity (Choudhuri \& Karak 2012). The third proposal is that the tidal influence of solar system planets may modulate the temperature gradient and consequently the magnetic storage capacity in the solar tachocline (Abreu et al. 2012). The first two mechanisms may account for the occurrence of Grand Minima, but fail to reproduce the observed long-term modulation of solar cycles. The third one, the planetary hypothesis (a long standing problem), has been intensively discussed in the last years by the scientific community (Charbonneau 2013), but has been criticized due to the inappropriate statistical analysis (Cameron \& Schüssler 2013; Poluianov \& Usoskin 2014) or data artifact (Cauquoin et al. 2014).

The long-term variation of solar activity is probably connected to the tachocline, a thin layer below the solar convection zone, which is a very important place for the solar dynamo and angular momentum redistribution (Spiegel \& Zahn 1992). The tachocline is believed to consist of a lower strongly stratified layer and an upper overshoot weakly stratified layer (Gilman 2000). The convective motions from the convection zone penetrate the upper overshoot layer, while the lower layer is much more stable, and the ordinary MHD shallow water equations of Gilman (2000) can be safely applied there.

The conservation of total vorticity in hydrodynamic shallow water equations leads to the appearance of Rossby or planetary waves in the rotating frame, which govern the large-scale dynamics of the Earth's atmosphere (and oceans) causing the formation of cyclones/anticyclones and hurricanes. Inclusion of horizontal magnetic field splits the ordinary hydrodynamic Rossby waves into fast and slow magnetic Rossby waves in a magnetized plasma (Zaqarashvili et al. 2007, 2009). Fast magnetic Rossby waves are responsible for the observed Rieger-type periodicity (155-160 days) in solar activity after the interaction with the dynamo magnetic field in the tachocline (Zaqarashvili et al. 2010). However, slow magnetic Rossby waves could have very long timescales compared to the solar rotation and solar cycle periodicity. In this Letter, we consider the slow magnetic Rossby modes in the lower strongly stable 
layer of the solar tachocline and show their connection with long-term variations in solar activity.

\section{SLOW MAGNETIC ROSSBY WAVES AND LONG PERIODS IN SOLAR ACTIVITY}

We consider the spherical coordinate system $(r, \theta, \phi)$, where $r$ is the radial coordinate, $\theta$ is the co-latitude, and $\phi$ is the longitude. The unperturbed magnetic field is assumed to be toroidal, i.e., $\boldsymbol{B}=B_{\phi}(\theta) \hat{\boldsymbol{e}}_{\phi}$. Then, the consideration of rigid rotation and a homogeneous magnetic field, $B_{\phi}=B_{0}=$ const, leads to the following dispersion relation for magnetic Rossby waves in the rotating frame (Zaqarashvili et al. 2007):

$$
\omega^{2}+\frac{2 \Omega_{0} m}{n(n+1)} \omega+\frac{B_{0}^{2} m^{2}}{4 \pi \rho R^{2}} \frac{2-n(n+1)}{n(n+1)}=0,
$$

where $\omega$ is the frequency of the spherical harmonic, $\Omega_{0}$ is the equatorial angular velocity, $R$ is the distance from the solar center, $\rho$ is the medium density, $m$ and $n$ are toroidal and poloidal wavenumbers, respectively. If $B_{0}^{2} /\left(4 \pi \rho \Omega_{0}^{2} R^{2}\right) \ll 1$, which is easily satisfied in the solar tachocline, then the low order spherical harmonics of slow magnetic Rossby modes are described by the dispersion relation

$$
\omega_{m n}=-m \Omega_{0} \frac{B_{0}^{2}}{4 \pi \rho \Omega_{0}^{2} R^{2}} \frac{2-n(n+1)}{2},
$$

where $\omega_{m n}$ is the frequency of the spherical harmonic. Each spherical harmonic with different $m$ and $n$ has a different frequency (and hence period) depending on the plasma parameters, mostly on the magnetic field strength, $B_{0}$.

In order to find the correlation between slow magnetic Rossby waves and the long-term variation in solar activity, the periods of spherical harmonics with different magnetic field strength should be compared to the timescales found in observational data. We performed a spectral analysis of yearly mean total sunspot number from 1700 to 2013, and found strong peaks at 100 and 180 years in the oscillation spectrum (Figure 1, upper panel). The periods can be identified with the well-known Gleissberg (Gleissberg 1939; Hathaway 2010) and Suess (Suess 1980) cycles, respectively. Longer oscillatory periods cannot be safely defined due to the short length of the data series ( $\sim 310$ years). To find longer periods, we used solar activity reconstructed from cosmogenic isotopes (Vonmoos et al. 2006; Usoskin et al. 2007) 10Be and 14C measured in the Greenland GRIP ice core and in tree rings, respectively, during the past 10 millennia. Both data sets show different periodicities (Usoskin et al. 2004; Steinhilber et al. 2012; Hanslmeier et al. 2013). In order to select reliable periods, we put forward a novel approach and overlapped the spectra of both data sets (Figure 1, lower panel). Then we selected four well defined periods of 1000, 500, 350, and 200 years in both data sets. 10Be data also show power at the periods of 700-800 years, but there are no corresponding periods in $14 \mathrm{C}$ data. Combining the power spectra of sunspot numbers and cosmogenic isotopes, we define the reliable periods in solar activity as $\sim 1000, \sim 500, \sim 350, \sim 200$, and $\sim 100$ years.

Using the plasma parameters at the tachocline $\left(\Omega_{0}\right.$ $\left.=2.7 \cdot 10^{-6} \mathrm{~s}^{-1}, \rho=0.2 \mathrm{~g} \mathrm{~cm}^{-3}, R=5 \cdot 10^{10} \mathrm{~cm}\right)$ and the magnetic field strength of $1200 \mathrm{G}$, we find that the periods of the modes $m=1, n=2,3,4,5,6$ as calculated from Equation $(2)$ are $\sim 1170, \sim 470, \sim 260, \sim 170$, and $\sim 110$ years,
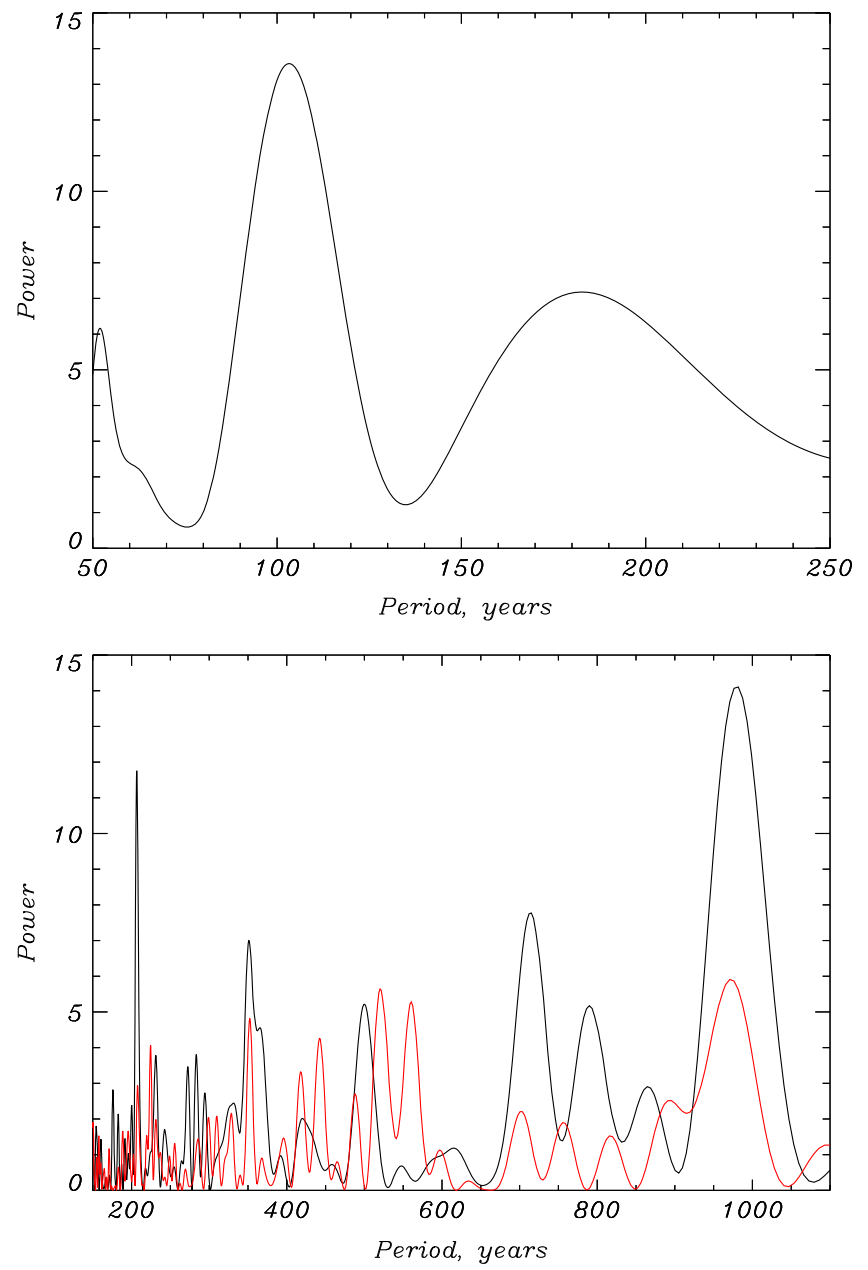

Figure 1. Upper panel: periodogram of yearly mean sunspot numbers. Large power values are concentrated around 180 and 100 years. Power is given in arbitrary units. Yearly mean sunspot data is downloaded from the World Data Center-SILSO, Royal Observatory of Belgium, Brussels. Lower panel: periodograms of reconstructed solar activity based on cosmogenic isotopes 10Be (Vonmoos et al. 2006; black line) and 14C (Usoskin et al. 2007; red line). Large power values in both data are concentrated around 1000, 500, 350, and 200 years. Power is given in arbitrary units.

respectively, which show perfect agreement between theoretical and observed periods (Figure 2). Therefore, we suggest that the observed periods in solar activity are the consequence of the first five spherical harmonics of slow magnetic Rossby modes in the lower tachocline.

The magnetic field strength of $1200 \mathrm{G}$, considered above, is much smaller than the estimated value of dynamo generated magnetic field in the tachocline $\left(10^{4}-10^{5} \mathrm{G}\right)$. Nevertheless, the toroidal component of the reversing dynamo field is zero when averaged over a time interval longer than the solar cycle length because of periodic sign reversals in consecutive cycles. Therefore, it probably cannot influence the long-period dynamics of slow magnetic Rossby waves. However, there are two different mechanisms, which may be responsible for the appearance of weak steady (nonreversing) toroidal magnetic field in the lower tachocline or upper radiative interior. First, Dikpati et al. (2006) showed that a steady (nonreversing) dynamo for a low enough magnetic diffusivity $\leqslant 10^{7} \mathrm{~cm}^{2} \mathrm{~s}^{-1}$ may generate steady toroidal magnetic field with the strength of $\sim 1 \mathrm{kG}$ in the lower tachocline. This is consistent to our requirements. Second, the primordial magnetic field, 


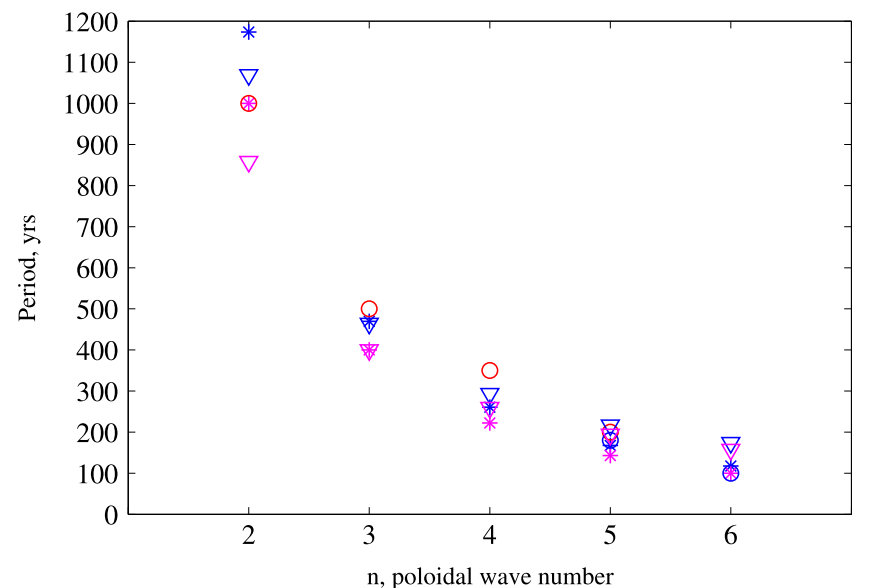

Figure 2. Periods of solar activity and slow magnetic Rossby modes with different poloidal wavenumber, $n$. Blue and red circles indicate the periods found by periodogram analysis of sunspot (Figure 1, upper panel) and radionuclide (Figure 1, lower panel) data, respectively. Blue and magenta asterisks indicate the periods of slow magnetic Rossby wave harmonics calculated from Equation (2) for $m=1$ and $n=2,3,4,5,6$ for magnetic field strength of 1200 and $1300 \mathrm{G}$, respectively. Blue and magenta triangles indicate the periods of the first five unstable harmonics calculated by the Legendre polynomial expansion for magnetic field strength of 1200 and $1300 \mathrm{G}$, respectively. Here $m=1$ and the differential rotation parameters are $s_{2}=s_{4}=0.01$.

which remained in the radiative interior after the Sun's collapse, may penetrate into the tachocline and affect Rossby waves. Both nonreversing dynamo and the primordial fields may be responsible for the observed Gnevyshev-Ohl 22 year rule (Gnevyshev \& Ohl 1948), which shows that odd sunspot cycles are generally stronger than even cycles, due to the break of polarity symmetry of the dynamo state (Boyer \& Levy 1984): the reversing dynamo magnetic field is aligned and antialigned with respect to the toroidal component of steady field in consecutive cycles leading to the consequence of strong and weak cycles, respectively. Similarly, the periodical variation caused by slow magnetic Rossby waves in the steady magnetic field can influence the strength of the dynamo magnetic field and consequently the cycle strength.

The primordial magnetic field strength was estimated as $1000 \mathrm{G}$ deep in the radiative interior and $1 \mathrm{G}$ just beneath the tachocline (Gough \& McIntyre 1998). This is the poloidal magnetic field, which probably has negligible influence on the cycle strength. Helioseismology shows that the radiative interior rotates almost uniformly; therefore, the magnetic field will remain poloidal in most of its part. On the other hand, the convection zone and the tachocline have differential rotation expressed as

$$
\Omega=\Omega_{0}\left(1-s_{2} \cos ^{2} \theta-s_{4} \cos ^{4} \theta\right),
$$

where $s_{2}$ and $s_{4}$ are parameters determined by observations whose values at the solar surface (and in the middle tachocline) are about 0.14. Schou et al. (1998) constructed (see Figure 7 in that paper) the radial dependence of solar rotation at different latitudes along the whole convection zone and radiative interior as inferred by helioseismology. The authors determined the center of the tachocline at $0.7-0.71 R_{\odot}$ with a negligible thickness of $0.05 R_{\odot}$; therefore, the lower boundary of the tachocline is located somewhere at $0.68 R_{\odot}$. A careful look at Figure 7 of Schou et al. (1998) shows that $\Omega$ is $445 \mathrm{nHz}$ at the equator and $415 \mathrm{nHz}$ at the latitude $60^{\circ}$ near the tachocline center $\left(0.7 R_{\odot}\right)$, which gives the differential rotation parameter $s_{2}=0.1$. This is close to the main value of differential rotation in the tachocline. On the other hand, $\Omega$ is $440 \mathrm{nHz}$ at the equator and $435 \mathrm{nHz}$ at the latitude $60^{\circ}$ near the lower boundary of the tachocline (at $0.68 R_{\odot}$ ), which gives $s_{2}=0.015$. Therefore, a small differential rotation is still present in the lower tachocline. This differential rotation may eventually lead to the generation of a toroidal component from the poloidal primordial field, which penetrates from the upper part of the radiative envelope into the lower part of the tachocline. The induction equation allows estimation of the generated toroidal field as $B_{\phi} / t_{0} \approx B_{\theta}(\partial \Omega / \partial \theta)$, where $t_{0}$ is the characteristic time (taken as the Alfvén time $R \sqrt{4 \pi \rho} / B_{\theta}$ ) and $B_{\theta}$ is the poloidal component of the primordial magnetic field. Then the toroidal magnetic field is

$$
B_{\phi} \sim \hat{B}_{0} \cos \theta \sin \theta
$$

where $\hat{B}_{0}$ is $4300 \mathrm{G}$ for $s_{2}=0.01$ and $1500 \mathrm{G}$ for $s_{2}=0.0035$. The strength of the estimated magnetic field coincides with the value required for slow magnetic Rossby waves to explain the observed periodicity. It should be noted that the equipartition value of steady toroidal magnetic field with the kinetic energy of turbulent pumping from the convection zone expressed as $B_{\text {eq }} \approx v \sqrt{4 \pi \rho}$, where $v$ is the turbulent velocity of convective cells $\left(0.01 \mathrm{~km} \mathrm{~s}^{-1}\right.$ near the base of the convection zone according to the standard mixing length theory of convection), is also estimated as $1500 \mathrm{G}$.

Therefore, the strength of the steady toroidal magnetic field in the lower tachocline generated either by steady dynamo for low magnetic diffusivity or by differential rotation acting on the primordial poloidal component can be safely taken as $1000-1500 \mathrm{G}$.

The solar cycle strength is defined by magnetic energy of dynamo layer; therefore, it is connected to the square of magnetic field strength. Longitudinally averaged magnetic energy of toroidal magnetic field is proportional to $\left\langle\left(B_{d}+B_{s}\right)^{2}\right\rangle_{\phi}=\left\langle B_{d}^{2}\right\rangle_{\phi}+\left\langle 2 B_{d} B_{s}\right\rangle_{\phi}+\left\langle B_{s}^{2}\right\rangle_{\phi}$, where brackets denote averaging over $\phi$ from 0 to $2 \pi, B_{d}$ is the dynamo generated reversing magnetic field, and $B_{s}$ is the steady nonreversing (primordial or steady dynamo) magnetic field. Spherical harmonics of slow magnetic Rossby waves lead to the toroidal dependence of steady magnetic field corresponding to the wavenumber, $m$. For example, $m=1$ mode leads to the dependence of $\cos \phi$ in the steady field. Then the longitudinally averaged magnetic energy is proportional to $B_{d}^{2}+B_{s 0}^{2} / 2$, where $B_{s 0}$ is the amplitude of steady field. $B_{s 0}$ has temporal variations due to the slow magnetic Rossby waves as discussed above; therefore, it can be responsible for the long period modulation of longitudinally averaged magnetic energy and hence the solar cycle strength.

The latitudinal differential rotation in the lower part of the tachocline (or in the upper part of the radiative interior), even if small, may lead to magnetohydrodynamic instabilities in joint action with the toroidal magnetic field (Gilman \& Fox 1997; Cally et al. 2003). In order to find unstable harmonics, we use the general technique of Legendre polynomial expansion (Longuet-Higgins 1968). 2D shallow water MHD equations in the presence of differential rotation (Equation (3)) and a 
toroidal magnetic field (Equation (4)) can be written in the rotating frame as (Zaqarashvili et al. 2010)

$$
\begin{gathered}
\left(\Omega_{d}-\hat{\omega}\right) L \Psi+\left(2-\frac{d^{2}}{d \mu^{2}}\left[\Omega_{d}\left(1-\mu^{2}\right)\right]\right) \Psi \\
-\mu \beta^{2} L \Phi-6 \mu \beta^{2} \Phi=0, \\
\left(\Omega_{d}-\hat{\omega}\right) \Phi=\mu \Psi,
\end{gathered}
$$

where

$$
L=\frac{\partial}{\partial \mu}\left(1-\mu^{2}\right) \frac{\partial}{\partial \mu}-\frac{m^{2}}{1-\mu^{2}}
$$

is the Legendre operator, $\Psi$ and $\Phi$ are the stream functions for velocity and magnetic field perturbations, $\mu=\cos \theta$, and

$$
\Omega_{d}(\mu)=-s_{2} \mu^{2}-s_{4} \mu^{4}, \hat{\omega}=\frac{\omega}{\Omega_{0}}, \beta^{2}=\frac{B_{0}^{2}}{4 \pi \rho \Omega_{0}^{2} R^{2}} .
$$

We expand $\Psi$ and $\Phi$ in infinite series of associated Legendre polynomials

$$
\Psi=\sum_{n=m}^{\infty} a_{n} P_{n}^{m}(\mu), \Phi=\sum_{n=m}^{\infty} b_{n} P_{n}^{m}(\mu),
$$

substitute them into Equations (5) and (6), and using a recurrence relation of Legendre polynomials, obtain algebraic equations as infinite series (Zaqarashvili et al. 2010). The dispersion relation for the infinite number of harmonics can be obtained when the infinite determinant of the system is set to zero. In order to solve the determinant, we truncate the series at $n=75$ and solve the resulting polynomial in $\omega$ numerically. The frequencies of different harmonics can be real or complex giving the stable or unstable character of a particular harmonic. Then differential rotation parameters $s_{2}=s_{4}=0.01$ (as estimated in the previous paragraph) and the magnetic field strength $B_{0}=1200-1300 \mathrm{G}$ lead to periods of unstable harmonics - which are close to the observationally obtained and analytically predicted values (Figure 2).

\section{DISCUSSION AND CONCLUSIONS}

It is shown in this Letter that the spherical harmonics of slow magnetic Rossby waves have very long periods in the presence of a steady toroidal magnetic field near the lower boundary of the tachocline. The steady toroidal field can be generated in the lower tachocline either due to a steady dynamo in the case of low magnetic diffusivity (Dikpati et al. 2006) or due to action of latitudinal differential rotation on poloidal primordial magnetic field. The strength of steady dynamo magnetic field in the lower tachocline is $\sim 1 \mathrm{kG}$ in the case of low magnetic diffusivity $\left(\leqslant 10^{7} \mathrm{~cm}^{2} \mathrm{~s}^{-1}\right.$; Dikpati et al. 2006). The poloidal primordial magnetic field may penetrate the lower part of the tachocline from the radiative interior and generate the toroidal component due to the small latitudinal differential rotation, which is present near the interface of the radiative interior and the tachocline (Schou et al. 1998). The differential rotation rate of the order of $s_{2}=0.003-0.01$ leads to the equipartition value of toroidal magnetic field of 1500-4000 G. In both cases of steady dynamo and primordial magnetic fields, the periods of spherical harmonics of slow magnetic Rossby waves correspond to the observed long periods in sunspot number

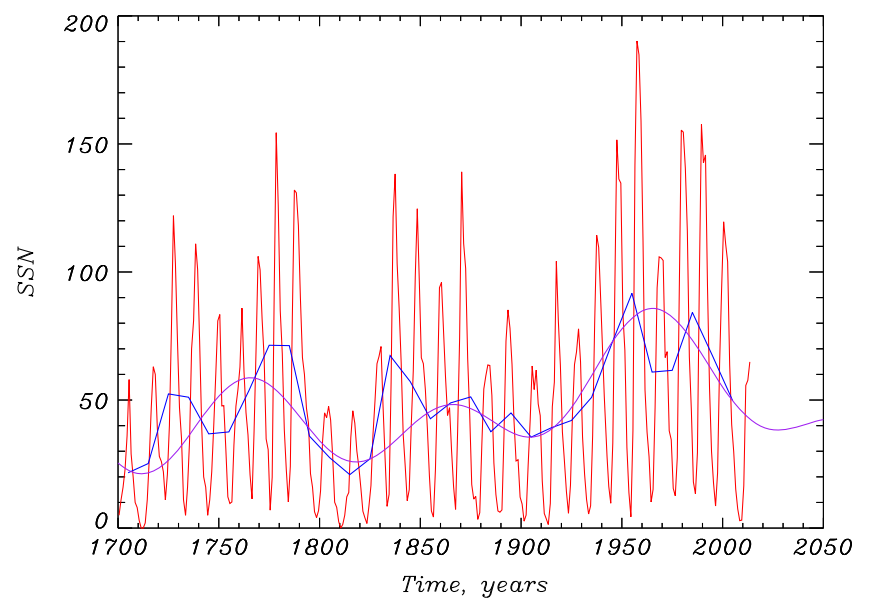

Figure 3. Forecast of future solar cycle strength using magnetic Rossby wave theory. Red and blue lines show yearly and 10 years averaged sunspot numbers, respectively. Magenta line shows the fit of the sum of 5 sinusoidal functions with periods of 1000, 500, 350, 200 and 100 years.

and radionuclide data. We believe that this coincidence has reliable physical ground: slow magnetic Rossby modes cause periodical variations of steady nonreversing toroidal magnetic field in the lower tachocline, which in turn modify the dynamo magnetic field and hence the solar cycle strength. The lower part of the tachocline is stable contrary to the upper overshoot layer as the convection does not penetrate there. Therefore, the steady toroidal magnetic field and large-scale slow magnetic Rossby waves may survive over a long time without significant influence.

MHD instabilities in the solar tachocline cause the appearance of active longitudes depending on the toroidal wavenumber, $m$ (Dikpati \& Gilman 2005). Then the magnetic Rossby waves may lead to the migration of active longitudes in the toroidal direction (Berdyugina \& Usoskin 2003; Gyenge et al. 2014). The phase speed of slow magnetic Rossby waves along the toridal direction is

$$
v_{\mathrm{ph}}=-\frac{B_{0}^{2}}{4 \pi \rho \Omega_{0} R} \frac{2-n(n+1)}{2}
$$

which for $n=2$ and $B_{0}=1200 \mathrm{G}$ has the value of $8 \mathrm{~cm} \mathrm{~s}^{-1}$. The speed is much slower compared to the surface speed of solar rotation; therefore, it is hard to catch the migration by observations.

Slow magnetic Rossby waves, which reproduce the observed long periods in solar activity, can be used for the long-term forecasting of the activity level. We fit the sum of five sinusoidal functions (with periods of 1000, 500, 350, 200, and 100 years), which are actually the spherical harmonics of slow magnetic Rossby modes, to the data of yearly mean total sunspot number from 1700 to 2013 (Figure 3). The resulting curve fits rather well the long-term behavior of the activity. Our model predicts the next deep minimum of solar activity around year 2030, which means that the next two solar cycles will be rather weak, but then the activity will start to increase again.

We also fit the sum of four sinusoidal functions $(1000,500$, 350 , and 200 years) to the 100 years averaged sunspot number for the last three millennia, reconstructed from $14 \mathrm{C}$ data (Figure 4). The fit is generally good except near the Spörer minimum, where a remarkable anti-phase relation is seen. This 


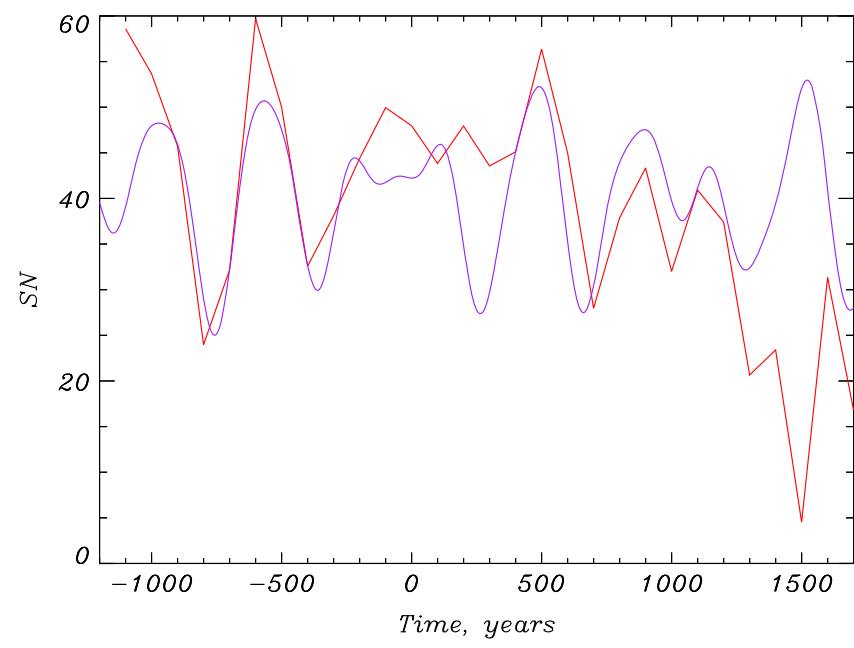

Figure 4. Fit of magnetic Rossby wave harmonics to reconstructed sunspot number for the last three millennia. Red line shows 100 year averaged sunspot number reconstructed from $14 \mathrm{C}$ data (Usoskin et al. 2014). Purple line shows the fit of the sum of 4 sinusoidal functions with periods of $1000,500,350$, and 200 years, which are the first four harmonics of slow magnetic Rossby waves.

disagreement is probably caused due to two distinct modes in solar activity as recently suggested by Usoskin et al. (2014).

The theory we have described provides a simple view of the coupling between the large-scale dynamics of the radiative interior and the tachocline. Dynamic interaction between rotation and steady (steady dynamo or primordial) toroidal magnetic field in the upper part of the radiative interior and the tachocline inevitably results in long periodicities due to slow magnetic Rossby modes, which accordingly modify the reversing dynamo magnetic field causing the observed longterm variations in solar activity. The scenario is a starting point for future sophisticated models with comprehensive study (including intense numerical simulations), which will enhance the accuracy of long-term solar activity forecast. The theory may also provide a hint for observed long-period modulations of magnetic activity in solar-like stars.

The work of T.Z. was supported by the Austrian Fonds zur Förderung der wissenschaftlichen Forschung under project P26181-N27, by FP7-PEOPLE-2010-IRSES-269299 projectSOLSPANET and by Shota Rustaveli Foundation grant DI/14/
6-310/12. I.G.U. acknowledges the ReSOLVE Centre of Excellence (Academy of Finland, project no 272157). Source of yearly mean sunspot numbers: WDC-SILSO, Royal Observatory of Belgium, Brussels.

\section{REFERENCES}

Abreu, J. A., Beer, J., Ferriz-Mas, A., McCracken, K. G., \& Steinhilber, F. 2012, A\&A, 548, A88

Berdyugina, S. V., \& Usoskin, I. G. 2003, A\&A, 405, 1121

Boyer, D. W., \& Levy, E. H. 1984, ApJ, 277, 848

Cally, P. S., Dikpati, M., \& Gilman, P. A. 2003, ApJ, 582, 1190

Cameron, R. H., \& Schüssler, M. 2013, A\&A, 557, A83

Cauquoin, A., Raisbeck, G. M., Jouzel, J., \& Bard, E. 2014, A\&A, 561, A132

Charbonneau, P. 2005, LRSP, 2, 2

Charbonneau, P. 2013, Natur, 493, 613

Choudhuri, A. R., \& Karak, B. B. 2012, PhRvL, 109, 171103

Dikpati, M., \& Gilman, P. A. 2005, ApJL, 635, L193

Dikpati, M., Gilman, P. A., \& MacGregor, K. B. 2006, ApJ, 638, 564

Haigh, J. D. 2007, LRSP, 4, 2

Hanslmeier, A., Brajša, R., Čalogović, J., et al. 2013, A\&A, 550, A6

Hathaway, D. H. 2010, LRSP, 7, 1

Hill, J. R. 1977, Natur, 266, 151

Gilman, P. A. 2000, ApJL, 544, L79

Gilman, P. A., \& Fox, O. A. 1997, ApJ, 484, 439

Gleissberg, M. N. 1939, Obs, 62, 158

Gnevyshev, M. N., \& Ohl, A. I. 1948, AZh, 25, 18

Gough, D. O., \& McIntyre, M. E. 1998, Natur, 394, 755

Gyenge, N., Baranyi, T., \& Ludmány, A. 2014, SoPh, 289, 579

Longuet-Higgins, M. S. 1968, Proc. R. Soc. London. A, 262, 511

Poluianov, S., \& Usoskin, I. G. 2014, SoPh, 289, 2333

Schou, J., Antia, H. M., Basu, S., et al. 1998, ApJ, 505, 390

Schwabe, H. 1844, AN, 21, 233

Solanki, S. K., Usoskin, I. G., Kromer, B., Schüssler, M., \& Beer, J. 2004, Natur, 431, 1084

Spiegel, E. A., \& Zahn, J.-P. 1992, A\&A, 265, 106

Steinhilber, F., Abreu, J. A., Beer, J., et al. 2012, PNAS, 109, 5967

Stuiver, M., \& Braziunas, T. F. 1989, Natur, 338, 405

Suess, H. E. 1980, Radiocarbon, 22, 200

Usoskin, I. G., Hulot, G., Gallet, Y., et al. 2014, A\&A, 562, L10

Usoskin, I. G., Mursula, K., Solanki, S., Schüssler, M., \& Alanko, K. 2004, A\&A, 413, 745

Usoskin, I. G., Solanki, S. K., \& Kovaltsov, G. A. 2007, A\&A, 471, 301

Vonmoos, M., Beer, J., \& Muscheler, R. 2006, JGRA, 111, A10105

Weiss, N. O., Cattaneo, F., \& Jones, C. A. 1984, GApFD, 30, 305

Zaqarashvili, T. V., Carbonell, M., Oliver, R., \& Ballester, J. L. 2010, ApJ, 709,749

Zaqarashvili, T. V., Oliver, R., \& Ballester, J. L. 2009, ApJL, 691, L41

Zaqarashvili, T. V., Oliver, R., Ballester, J. L., \& Shergelashvili, B. M. 2007, A\&A, 470, 815 